\title{
Platelet-Oriented Inhibition in New TIA and Minor Ischemic Stroke (POINT) Trial: Rationale and design
}

\author{
S. Claiborne Johnston 1,2*, J. Donald Easton ${ }^{1}$, Mary Farrant ${ }^{1}$, William Barsan ${ }^{3}$, \\ Holly Battenhouse ${ }^{4}$, Robin Conwit ${ }^{5}$, Catherine Dillon ${ }^{4}$, Jordan Elm ${ }^{5}$, Anne Lindblad ${ }^{6}$, \\ Lewis Morgenstern ${ }^{3}$, Sharon N. Poisson ${ }^{7}$, and Yuko Palesch ${ }^{5}$
}

\begin{abstract}
Background Ischemic stroke and other vascular outcomes occur in $10-20 \%$ of patients in the three-months following a transient ischemic attack or minor ischemic stroke, and many are disabling. The highest risk period for these outcomes is the early hours and days immediately following the ischemic event. Aspirin is the most common antithrombotic treatment used for these patients.

Aim The aim of POINT is to determine whether clopidogrel plus aspirin taken $<12 \mathrm{~h}$ after transient ischemic attack or minor ischemic stroke symptom onset is more effective in preventing major ischemic vascular events at 90 days in the high-risk, and acceptably safe, compared with aspirin alone.
\end{abstract}

Correspondence: S. Claiborne Johnston*, UCSF Department of

Neurology, UCSF Clinical and Translational Science Institute, Sandler

Neurosciences Bldg., 675 Nelson Rising Lane, San Francisco, CA

94158-0663, USA.

E-mail: clay.johnston@ucsfmedctr.org

${ }^{1}$ Department of Neurology, University of California, San Francisco, CA, USA

${ }^{2}$ Department of Epidemiology and Biostatistics, University of California, San Francisco, CA, USA

${ }^{3}$ Emergency Medicine, University of Michigan Health System, Ann Arbor, MI, USA

${ }^{4}$ Data Coordination Unit, Department of Public Health Services, Medical University of South Carolina, Charleston, SC, USA

${ }^{5}$ Office of Clinical Research, National Institute of Neurological Disorders, Bethesda, MD, USA

${ }^{6}$ The EMMES Corporation, Rockville, MD, USA

${ }^{7}$ Department of Neurology, University of Colorado School of Medicine, Denver, CO, USA

Disclosure: S. C. Johnston is funded by NIH grants 1U01 NS062835 and UL1 RR024131, has received research support from the American Heart Association, is coeditor of Journal Watch Neurology and vice editor of the Annals of Neurology, is a coholder of a patent on an RNA panel to identify and risk-stratify transient ischemic attacks, indirectly receives research support from Sanofi (drug and placebo for this NIH-sponsored trial), and receives research support from AstraZeneca. J. D. Easton is funded by NIH grant 1U01 NS062835, is a consultant for Bristol-Myers Squibb; receives research support from AstraZeneca, and is on data monitoring noards for Daiichi-Sankyo, Schering-Plough Research Institute, Novartis, Cerevast Therapeutics, Acorda Therapeutics, and Johnson and Johnson, Inc. William Barsan is funded by NIH grants 2U01NS056975-06, 2U01NS056975-06, U01NS073476-01, U01 NS 062835, 1U01NS062778, and 1-U01-NS-069498-01. Anne Lindblad is an owner of The EMMES Corporation and through her employment at EMMES is supported by grants and contracts from the DoD, NEI, NINDS, and Acucela. Lewis Morgenstern is funded by NIH grants U01 NS062835, R01 HL098065, R01 NS070941, R18 HS017690, R01 NS38916, R01 NS062675, and U01 NS056975. Mary Farrant, Holly Battenhouse, Robin Conwit, Catherine Dillon, Jordan Elm, Sharon N. Poisson, and Yuko Palesch declare no funding or potential conflict of interest.

Clinical Trial Registration - URL: http://www.clinicaltrials.gov Unique identifier: NCT00991029.

DOI: $10.1111 /$ ijs.12129
Design POINT is a prospective, randomized, double-blind, multicenter trial in patients with transient ischemic attack or minor ischemic stroke. Subjects are randomized to clopidogrel (600 mg loading dose followed by $75 \mathrm{mg} /$ day) or matching placebo, and all will receive open-label aspirin 50-325 mg/day, with a dose of $162 \mathrm{mg}$ daily for five-days followed by $81 \mathrm{mg}$ daily strongly recommended.

Study Outcomes The primary efficacy outcome is the composite of new ischemic vascular events - ischemic stroke, myocardial infarction, or ischemic vascular death - by 90 days. The primary safety outcome is major hemorrhage, which includes symptomatic intracranial hemorrhage.

Discussion Aspirin is the most common antithrombotic given to patients with a stroke or transient ischemic attack, as it reduces the risk of subsequent stroke. This trial expects to determine whether more aggressive antithrombotic therapy with clopidogrel plus aspirin, initiated acutely, is more effective than aspirin alone.

Key words: aspirin, clinical trial, clopidogrel, minor stroke, stroke prevention, TIA

\section{Introduction and rationale}

Transient ischemic attacks (TIAs) are common and often harbingers of disabling ischemic strokes. Between 200000 and 500000 TIAs are diagnosed each year in the United States $(1,2)$, while approximately 795000 patients experience a new or recurrent stroke (3).

Rapid recovery from cerebral ischemia is a defining characteristic of TIA and distinguishes it from completed stroke $(1,4-9)$. This recovery delineates a distinct pathophysiologic feature that generally indicates the presence of previously ischemic tissue still at risk, a characteristic that may be responsible for greater instability $(9,10)$. The same is true for patients with minor ischemic strokes. The distinction between minor ischemic stroke and TIA is unimportant in terms of prognosis, as both groups are at high short-term risk for new, and often disabling, ischemic stroke and other vascular events (11-13). These serious vascular outcomes occur in $10-20 \%$ of patients in the three-months following a TIA or minor ischemic stroke, and the highest risk period for these outcomes is the early hours and days immediately following the ischemic event (11-13). Platelet aggregation is an important contributing factor in cerebral ischemia, as in other forms of atherothrombotic ischemia. Antiplatelet drugs reduce the risk of ischemic stroke in this setting. Aspirin is the most common antithrombotic given to patients with a history of stroke or TIA, as it reduces the risk of subsequent stroke. More aggressive antithrombotic therapy, especially dual antiplatelet drugs, in patients with prior ischemic stroke is commonly associated with a higher risk of intracranial and other major hemorrhages (14-20). Yet more 
aggressive therapies may be effective in TIA and minor stroke patients who are at high short-term risk for thrombosis and have minimal ischemic brain damage that could increase their risk for bleeding. Aggressive antithrombotic therapy could significantly reduce the overall burden of stroke if initiated immediately. The Fast Assessment of Stroke and Transient Ischaemic Attack to Prevent Early Recurrence (FASTER) pilot trial (21) showed a trend toward benefit for this approach. The Clopidogrel in Highrisk patients with Acute Non-disabling Cerebrovascular Events (CHANCE) trial is a large-scale trial evaluating an acute intervention in patients with TIA or nondisabling stroke (22), and the results should be published soon.

The purpose of the Platelet-Oriented Inhibition in New TIA and Minor Ischemic Stroke (POINT) study is to determine whether clopidogrel, when initiated acutely, is more effective than placebo in preventing major ischemic vascular events in patients with TIA or minor ischemic stroke, on a background of aspirin. This article describes the POINT trial design.

\section{Methods}

\section{Design and patient population}

The design of POINT is shown in Fig. 1. The trial enrolls subjects 18 years of age or older with high-risk TIAs, defined as an $\mathrm{ABCD}^{2}$ (23) score $\geq 4$, or minor ischemic stroke, with a National Institutes of Health Stroke Scale (24) score $\leq 3$, who can be randomized within $12 \mathrm{~h}$ of the time last known free of new ischemic symptoms. Table 1 lists the inclusion and exclusion criteria.

The study is conducted in accordance with the U.S. Food and Drug Administration (FDA)'s Code of Federal Regulations (CFR 21) and Good Clinical Practice guidelines.

Prior to initiating the study, each site obtains institutional review board (IRB) approval for the protocol, informed consent documents, and materials used to recruit subjects. All changes to the protocol are submitted to each site's IRB for review and approval as appropriate. The trial has received an Investigational New Drug (IND) application waiver from the FDA.

Each eligible patient who wishes to participate is required to give written informed consent. The consent document explains the risks and potential benefits of the therapy, the procedures for the trial, and alternatives to participation. In addition, a video explanation of the study on an iPad will be provided for potential enrollees. There is no surrogate consent in the study.

The maximum total sample size for the study is 4150 subjects from a total of up to 210 investigational sites in the United States and worldwide.

Face-to-face study visits occur on the day of randomization, after a possible stroke or myocardial infarction (MI), and at Day 90. Visits are encouraged when serious adverse events occur. Telephone calls occur on Days 7 and 30. Details of the information collected are available in Supporting Information Table S1 in the online version of this article.

\section{Randomization}

Randomization takes place centrally and electronically via the WebDCU $^{\text {тм }}$ clinical trials management system housed at the POINT Statistics and Data Coordinating Center at the Medical University of South Carolina. Subjects are randomized 1:1 (clopidogrel:placebo), treatments balanced within clinical centers using the blocked-urn method. The randomization computer program makes the treatment assignment based on the current status of treatment group distribution within each clinical center as well as overall balance of treatment assignment.

\section{Treatment}

Patients will be randomized into two groups. The first group will receive a 600 -mg loading dose of clopidogrel, followed by $75 \mathrm{mg} /$ day from Day 2 to Day 90. The second group will receive eight placebo tablets, matched in appearance and taste to the clopi-

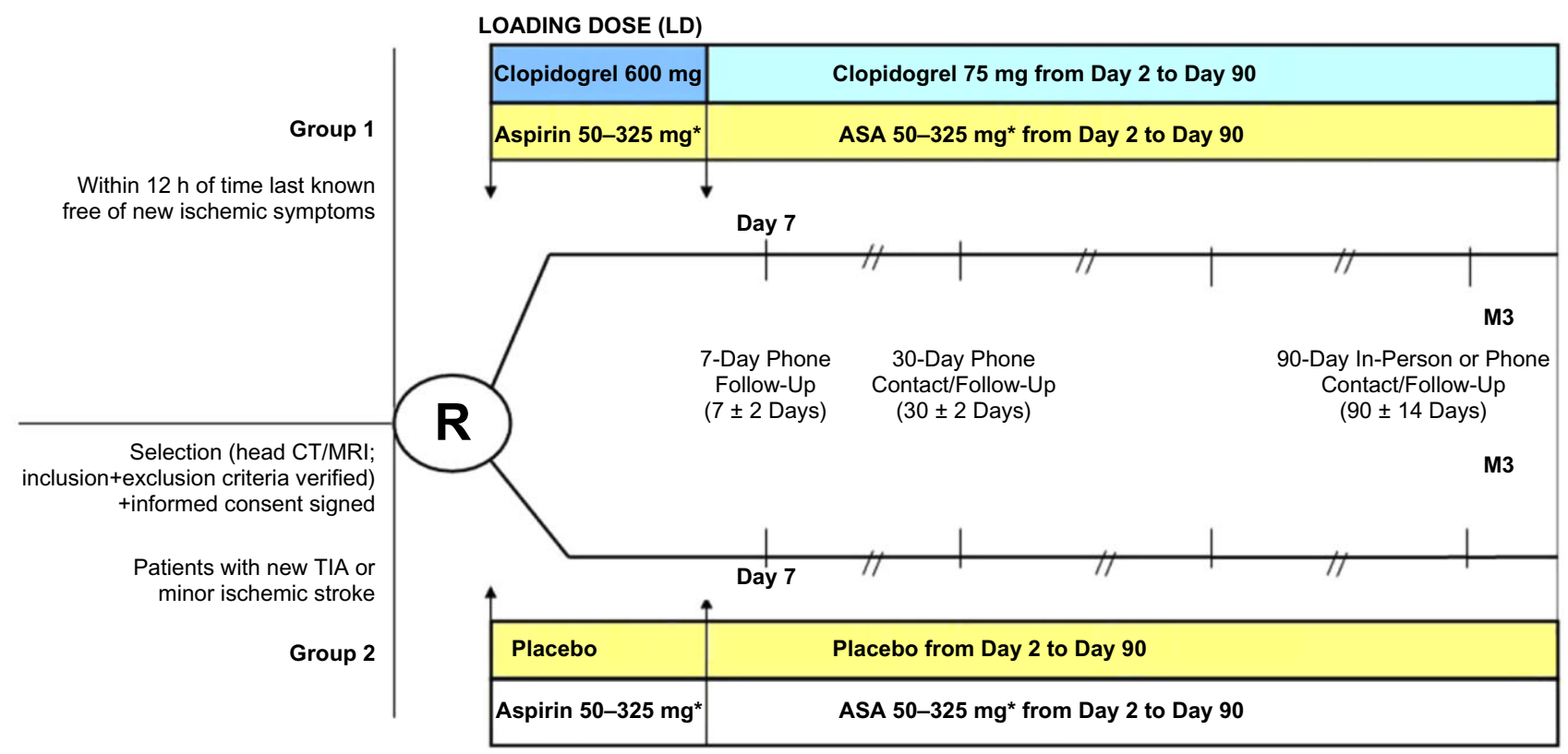

Fig. 1 Study flowchart. *Open-label aspirin (at the discretion of the investigator) with dose of $162 \mathrm{mg}$ daily for five-days, followed by $81 \mathrm{mg}$ daily for the remaining 85 days, strongly recommended. 
Table 1 Inclusion and exclusion criteria

Inclusion criteria

- Neurologic deficit (based on history or examination) attributed to focal brain ischemia and either:

o High-risk transient ischemic attack: Complete resolution of the deficit at the time of randomization and $A B C D^{2} s c o r e \geq 4$

or

o Minor ischemic stroke: residual deficit with National Institutes of Health Stroke Scale score $\leq 3$ at the time of randomization

- Ability to randomize within $12 \mathrm{~h}$ of time last known free of new ischemic symptoms.

- Computed tomography or magnetic resonance imaging of the head ruling out hemorrhage or other pathology, such as vascular malformation, tumor, or abscess, that could explain symptoms or contraindicate therapy.

- Ability to tolerate aspirin at a dose of 50-325 mg/day.

Exclusion criteria

- Age $<18$ years

- Transient ischemic attack symptoms limited to isolated numbness, isolated visual changes, or isolated dizziness/vertigo.

- In the judgment of the treating physician, a candidate for thrombolysis, endarterectomy or endovascular intervention, unless the subject declines both endarterectomy and endovascular intervention at the time of evaluation for eligibility.

- Receipt of any intravenous or intra-arterial thrombolysis within one-week prior to index event.

- Gastrointestinal bleed or major surgery within three-months prior to index event.

- History of nontraumatic intracranial hemorrhage.

- Clear indication for anticoagulation (e.g., warfarin, heparin) anticipated during the study period (atrial fibrillation, mechanical heart valve, deep venous thrombosis, pulmonary embolism, antiphospholipid antibody syndrome, hypercoagulable state).

- Qualifying ischemic event induced by angiography or surgery.

- Severe noncardiovascular comorbidity with life expectancy less than three-months.

- Contraindication to clopidogrel or aspirin:

- Known allergy to clopidogrel or aspirin

- Severe renal (serum creatinine $>2 \mathrm{mg} / \mathrm{dl}$ ) or hepatic insufficiency (prior or concurrent diagnosis, with International Normalized Ratio >1.5, or any resultant complication, such as variceal bleeding, encephalopathy, or icterus)

- Hemostatic disorder or systemic bleeding in the past three-months

- Current thrombocytopenia (platelet count $\left.<100 \times 10^{9} / \mathrm{l}\right)$ or neutropenia/granulocytopenia $\left(<1 \times 10^{9} / \mathrm{I}\right)$

- History of drug-induced hematologic or hepatic abnormalities

- Anticipated requirement for long-term (>7 days) nonstudy antiplatelet drugs (e.g., dipyridamole, clopidogrel, ticlopidine) or nonsteroidal anti-inflammatory drugs affecting platelet function (such as prior vascular stent or arthritis).

- Not willing or able to discontinue prohibited concomitant medications.

- Inability to swallow medications.

- At risk for pregnancy: premenopausal or postmenopausal woman within 12 months of last menses without a negative pregnancy test or not committing to adequate birth control (e.g., oral contraceptive, two methods of barrier birth control, or abstinence).

- Unavailability for follow-up.

- Signed and dated informed consent not obtained from patient.

- Other neurological conditions that would complicate assessment of outcomes during follow-up.

- Ongoing treatment in another study of an investigational therapy, or treatment in such a study within the last 7 days.

- Previously enrolled in the POINT study.

dogrel tablets, followed by one placebo tablet daily from Day 2 to Day 90. Both groups will be given open-label aspirin 50-325 mg/ day: a dose of $162 \mathrm{mg}$ daily for five-days, then the recommended $81 \mathrm{mg}$ daily dose. The first dose of study medication is given as soon after randomization as possible but no later than $12 \mathrm{~h}$ from symptom onset. Each subject is followed for 90 days from randomization. The trial is expected to complete in seven-years.

\section{Outcomes}

The primary efficacy outcome is the composite of new ischemic vascular events: ischemic stroke, MI, or ischemic vascular death, up to 90 days. POINT uses the tissue-based definition of stroke and TIA (25). If a subject has rapid resolution of symptoms and no brain imaging suggesting tissue infarction, he/she is considered to have had a TIA. Any brain imaging evidence of infarction or clinical evidence (such as symptoms persisting beyond $24 \mathrm{~h}$ ) qualifies the event as a stroke. Any patient initially diagnosed with stroke who does not have further brain imaging with evidence of infarction but has complete resolution of symptoms within $24 \mathrm{~h}$ is considered to have had a TIA.
It often is difficult to determine whether new neurologic deficits that develop after enrollment are due to stroke recurrence or to stroke progression or infarct growth (26). As the likely pathophysiology of these events is additional thrombosis, potentially amenable to prompt antithrombotic treatment, POINT does not distinguish between recurrence and progression.

The primary safety outcome is major hemorrhage. The definition of major bleeding is adapted from the protocol and the International Society on Thrombosis and Hemostasis (27) and Prevention Regimen for Effectively Avoiding Second Strokes (PRoFESS) Trial (18) definitions. Major hemorrhage is one that results in symptomatic intracranial hemorrhage, intraocular bleeding causing loss of vision, need for transfusion of two or more units of red cells or equivalent amount of whole blood, need for hospitalization or prolongation of an existing hospitalization, or death. This may include bleeding events related to surgical procedures. The adjudication committee evaluates all components of the primary efficacy and safety outcomes. 
Several secondary analyses will be performed as described under Statistical Considerations, including death (all causes), intracerebral hemorrhage, and minor hemorrhage.

\section{Data safety and monitoring board}

The National Institute of Neurological Disorders and Stroke (NINDS) appointed a Data Safety Monitoring Board (DSMB) as advisory to the NINDS. The NINDS is responsible for reviewing the DSMB recommendations to decide whether to continue or terminate the trial and to determine whether amendments to the protocol or changes in study conduct are required.

\section{Sample size}

The maximum total sample size for the study is 4150 subjects. With a sample size of 4150 patients, with 530 events, the study has $90 \%$ power to detect a relative risk reduction of $23 \%$ with a two-sided alpha of $0 \cdot 05$. The sample size was estimated based on a hazard ratio (HR) of 0.75 (equivalent to relative risk reduction of $23 \%$ ) under the exponential survival distribution (assuming the proportion of patients with events in the placebo group is $15 \%$ at Day 90), with inflation to account for two interim analyses for efficacy using O'Brien-Fleming-type stopping boundaries. The intent-to-treat principle will be applied to the primary outcome analysis, and therefore, the sample size was inflated to safeguard against 5\% lost to follow-up and/or crossover in the actual treatment received, which may dilute the effect size.

\section{Statistical analyses}

Complete details can be found in the Statistical Analysis Plan (SAP), available in the Supporting Information of the online version of this article. A final SAP will be issued by the Statistical and Data Management Center (SDMC) prior to database lock and before code breaking. The SAP defines all 'pre-specified, planned analyses'.

The primary null hypothesis of the trial is that in subjects with TIA or minor ischemic stroke treated with aspirin $50-325 \mathrm{mg} /$ day, there is no difference in event-free survival at the Day 90 follow-up in those treated with clopidogrel (600 mg loading dose, then $75 \mathrm{mg} /$ day) compared with placebo when subjects are randomized within 12 hours of time last known free from new ischemic symptoms. The primary outcome is a composite consisting of ischemic stroke, MI, or ischemic vascular death. The primary efficacy hypothesis is tested with the log-rank test for equality of survival curves. This hypothesis is tested with a two-sided level of significance of $0 \cdot 05$. The primary analysis is intention-to-treat, with inclusion and treatment group defined per the randomization assignment. Subjects missing Day 90 follow-up visits are censored at the last follow-up assessment date. The primary safety outcome is major hemorrhage.

A number of other secondary outcomes are planned to be evaluated separately, including risk of ischemic stroke, all-cause mortality, intracranial hemorrhage and major hemorrhage, and the composite of the primary outcome and major hemorrhage. The influence of index event type (TIA vs. minor stroke), gender, and race/ethnicity will be evaluated in subgroup analyses.

The analysis strategy outlined for the primary outcome will be used for most of the secondary analyses. Secondary analyses will be tested at the two-sided alpha of 0.05 without accounting for multiplicity. These analyses will be viewed as exploratory hypotheses that may or may not support the results of the primary analysis.

After one-third and two-thirds of events occur, formal interim analyses of the primary outcome will be conducted to consider stopping the trial for overwhelming efficacy or for futility. The criterion for determination of futility is as follows: if the conditional power (defined as the probability of rejecting the null hypothesis at the final analysis given the data accumulated so far) falls below 20\%, then the DSMB evaluates all study information (such as overall recruitment rate and secondary outcome assessment data) to consider stopping the study for futility. Conditional power is computed under two assumptions: assuming the alternative is true and assuming the current trend is true. At the time of the first interim analysis, the sample size may be re-estimated solely based on the placebo event rate. If the one-sided upper $99 \%$ confidence limit around the observed placebo rate does not overlap with those of the assumed rate (15.24\%; 95\% CI $13 \cdot 63-$ $16.85 \%$ ) based on a sample of 1907 TIA patients from Kaiser-Permanente Northern California (KPNC) (12), then the maximum sample size will be re-estimated based on the observed placebo event rate (assuming the original treatment effect, $\mathrm{HR}=0 \cdot 75)$.

\section{Study organization and funding}

The principal investigator, who has ultimate responsibility for all activities and products of the trial and oversees all functions, directs the trial.

The trial management is a partnership of the University of California, San Francisco Clinical Coordinating Center (UCSF CCC) based in the Stroke Sciences Group; the NINDS Neurological Emergencies Treatment Trials (NETT) Network, which is responsible for statistical analyses, data management and oversight of NETT sites; and the POINT Clinical Research Collaboration (CRC), supported by The EMMES Corporation, which manages the non-NETT sites, including the sites outside the United States.

The NINDS NETT Network, through its SDMC, provides statistical support and data management services, including reports to the DSMB and Clinician Events Coordinator, and shielding of the UCSF CCC, NETT-CCC, and POINT CRC from access to unblinded data during the trial. The director of the NETT SDMC is responsible for the randomization protocol, statistical analysis plan, and final data analysis.

The NINDS funds the POINT. Sanofi contributes clopidogrel and its placebo at no cost and with no restrictions. The principal investigator and executive committee will have full access to the entire dataset at trial completion and are responsible for analysis and publication in collaboration with the sponsor.

\section{Discussion}

People who experience a TIA or minor ischemic stroke are at substantial risk for a major stroke and other ischemic events in the subsequent hours and days. They are most often given aspirin for anti-atherothrombotic treatment. Others are treated with clopi- 
dogrel or aspirin plus dipyridamole. Recently, more of these patients are treated with clopidogrel plus aspirin for several days or weeks, especially if they experience a cerebral ischemic event while on aspirin. It is not known whether this latter treatment is efficacious or safe. The POINT trial will evaluate whether clopidogrel plus aspirin is more effective, and acceptably safe, than aspirin alone in preventing ischemic stroke and other vascular outcomes in the high-risk period after TIA or minor ischemic stroke, when the risk for thrombosis is high and the risk for serious hemorrhage should be low. Whether the trial result is positive or negative, it will provide valuable clarification of the appropriate treatment for these patients in whom the best treatment is currently uncertain.

\section{Statements}

The authors are solely responsible for the design and conduct of this study, study analyses, and the drafting and editing of the paper.

\section{References}

1 Johnston SC, Fayad PB, Gorelick PB et al. Prevalence and knowledge of transient ischemic attack among US adults. Neurology 2003; 60:1429-34.

2 Kleindorfer D, Panagos P, Pancioli A et al. Incidence and short-term prognosis of transient ischemic attack in a population-based study. Stroke 2005; 36:720-3.

3 Writing Group Members, Roger VL, Go AS et al. Heart disease and stroke statistics-2012 update. Circulation 2012; 125:e2-e220.

4 Alexandrov AV, Felberg RA, Demchuk AM et al. Deterioration following spontaneous improvement : sonographic findings in patients with acutely resolving symptoms of cerebral ischemia. Stroke 2000; 31:915-9.

5 Aslanyan S, Weir CJ, Johnston CS, Krams M, Grieve AP, Lees KR. The association of post-stroke neurological improvement with risk of subsequent deterioration due to stroke events. Eur J Neurol 2007; 14:1-6.

6 Aslanyan S, Weir CJ, Johnston SC, Lees KR. Poststroke neurological improvement within 7 days is associated with subsequent deterioration. Stroke 2004; 35:2165-70.

7 Grotta JC, Welch KM, Fagan SC et al. Clinical deterioration following improvement in the NINDS rt-PA Stroke Trial. Stroke 2001; 32:661-8.

8 Howard G, Toole JF, Frye-Pierson J, Hinshelwood LC. Factors influencing the survival of 451 transient ischemic attack patients. Stroke 1987; 18:552-7.

9 Johnston SC, Leira EC, Hansen MD, Adams HP, Jr. Early recovery after cerebral ischemia: risk of subsequent neurological deterioration. Ann Neurol 2003; 54:439-44.

10 Johnston SC, Easton JD. Are patients with acutely recovered cerebral ischemia more unstable? Stroke 2003; 34:2446-50.

11 Giles MF, Rothwell PM. Risk of stroke early after transient ischaemic attack: a systematic review and meta-analysis. Lancet Neurol 2007; 6:1063-72.

12 Johnston SC, Gress DR, Browner WS, Sidney S. Short-term prognosis after emergency department diagnosis of TIA. JAMA 2000; 284:2901-6.

13 Rothwell PM, Warlow CP. Timing of TIAs preceding stroke: time window for prevention is very short. Neurology 2005; 64:817-20.
14 Antman EM, Wiviott SD, Murphy SA et al. Early and late benefits of prasugrel in patients with acute coronary syndromes undergoing percutaneous coronary intervention: a TRITON-TIMI 38 (TRial to Assess Improvement in Therapeutic Outcomes by Optimizing Platelet InhibitioN with Prasugrel-Thrombolysis In Myocardial Infarction) analysis. J Am Coll Cardiol 2008; 51:2028-33.

15 Bhatt DL, Flather MD, Hacke W et al. Patients with prior myocardial infarction, stroke, or symptomatic peripheral arterial disease in the CHARISMA trial. J Am Coll Cardiol 2007; 49:1982-8.

16 Diener HC, Bogousslavsky J, Brass LM et al. Management of Atherothrombosis with Clopidogrel in High-Risk Patients with Recent Transient Ischaemic Attack or Ischaemic Stroke (MATCH): study design and baseline data. Cerebrovasc Dis 2004; 17:253-61.

17 Morrow DA, Braunwald E, Bonaca MP et al. Vorapaxar in the secondary prevention of atherothrombotic events. N Engl J Med 2012; 366:1404-13.

18 Sacco RL, Diener HC, Yusuf S et al. Aspirin and extended-release dipyridamole versus clopidogrel for recurrent stroke. $N$ Engl J Med 2008; 359:1238-51.

19 The ACTIVE Investigators. Effect of clopidogrel added to aspirin in patients with atrial fibrillation. N Engl J Med 2009; 360:2066-78.

20 The SPS3 Investigators. Secondary Prevention of Small Subcortical Strokes Trial (SPS3). ClinicalTrials.gov Identifier: NCT00059306; 2011.

21 Kennedy J, Hill MD, Ryckborst KJ, Eliasziw M, Demchuk AM, Buchan AM. Fast Assessment of Stroke and Transient Ischaemic Attack to Prevent Early Recurrence (FASTER): a randomised controlled pilot trial. Lancet Neurol 2007; 6:961-9.

22 Wang Y, Johnston SC. Rationale and design of a randomized, doubleblind trial comparing the effects of a 3-month clopidogrel-aspirin regimen versus aspirin alone for the treatment of high-risk patients with acute nondisabling cerebrovascular event. Am Heart J 2010; 160:380-6. e1.

23 Johnston SC, Rothwell PM, Nguyen-Huynh MN et al. Validation and refinement of scores to predict very early stroke risk after transient ischaemic attack. Lancet 2007; 369:283-92.

24 Lyden P, Brott T, Tilley B et al. Improved reliability of the NIH Stroke Scale using video training. NINDS TPA Stroke Study Group. Stroke 1994; 25:2220-6.

25 Easton JD, Saver JL, Albers GW et al. Definition and evaluation of transient ischemic attack: a scientific statement for healthcare professionals from the American Heart Association/American Stroke Association Stroke Council; Council on Cardiovascular Surgery and Anesthesia; Council on Cardiovascular Radiology and Intervention; Council on Cardiovascular Nursing; and the Interdisciplinary Council on Peripheral Vascular Disease. The American Academy of Neurology affirms the value of this statement as an educational tool for neurologists. Stroke 2009; 40:2276-93.

26 Coutts SB, Hill MD, Campos CR et al. Recurrent events in transient ischemic attack and minor stroke: what events are happening and to which patients? Stroke 2008; 39:2461-6.

27 Schulman S, Kearon C. Definition of major bleeding in clinical investigations of antihemostatic medicinal products in non-surgical patients. J Thromb Haemost 2005; 3:692-4.

\section{Supporting Information}

Additional Supporting Information may be found in the online version of this article at the publisher's web-site:

Table S1 Schedule of activities and assessments. 